\title{
Outcome of HER2 Testing by FISH applying ASCO/CAP 2007 and 2013 guideline in IHC equivocal group of breast cancer: Experience at tertiary cancer care centre
}

\author{
Manoj Kumar Panigrahi, Dushyant Kumar,Anurag Mehta', Kandarpa Kumar Saikia
}

\begin{abstract}
Background and Objectives: HER2 testing guideline of ASCO/CAP for interpretation and reporting has recently been revised. The study is aimed to measure the impact of 2013 CAP guideline on equivocal HER2 test outcome (immunohistochemistry [IHC] 2+) when tested by fluorescent in situ hybridization (FISH). The study also aims at finding the frequency of polysomy and monosomy of chromosome 17. Materials and Methods: Specimens were collected in Rajiv Gandhi Cancer Institute and Research Centre, New Delhi, India. IHC was performed in every case, and FISH was performed in IHC2+ cases. Results: In final analysis includes 557 subjects on the basis of CAP guideline 2007 and CAP guideline 20I3. One hundred ninety-two subjects (34.4\%) were HER2 amplified according to CAP scoring 2007, and 246 subjects (44\%) according to 20 I 3 CAP scoring. Conclusions: FISH results were evaluated (IHC2 + interpreted according to CAP 2007 guideline) with both 2007 and 2013 ASCO/CAP scoring criteria, we identified significantly more HER2 positive cases as compared to cases evaluated using the 2007 criteria $(P<0.05)$. We also found that in breast carcinoma, HER2 status in the presence of polysomy 17 may vary with the scoring criteria used. Evaluation of FISH result using 20I3 ASCO/CAP criteria means that more patients with breast cancer may be appropriate for targeted treatment with trastuzumab, potentially improving their outcome.
\end{abstract}

Key words: Fluorescent in situ hybridization technique, HER2 gene amplification, immunohistochemistry

\section{Introduction}

Breast cancer is the second most common cancer for women in the India. ${ }^{[1]}$ Researches have shown that about $20-30 \%$ of the breast cancer patients have HER2 amplification or overexpression that is associated with a more aggressive phenotype and decreased survival. ${ }^{[2]}$ Fluorescent in situ hybridization (FISH) is the gold standard because it was the strategy used during clinical trials and has proven clinical utility. Polysomy 17 indicates the presence of more than two copies of chromosome 17 and is reported in 13-46\% breast carcinoma. ${ }^{[3]}$ Amplification is by far the most common mechanism generating excess HER2 gene copies, polysomy occurs frequently. This has raised the question of whether increased HER2 protein expression can result not only from gene amplification but also from increased number of chromosome 17. The consequence would then be that patients with polysomy 17 tumors might be candidates for trastuzumab. ${ }^{[4]}$

This study includes 557 breast cancer patients with equivocal HER2 test results by immunohistochemical staining (immunohistochemistry [IHC] 2+) with an aim to measure the impact of changed criteria of ASCO/CAP recommendation 2007 and 2013 as well as frequency of polysomy 17 and deletion of HER2 gene.

\section{Materials and Methods}

\section{Sample collection and fixation protocol}

Five hundred and fifty-seven cases with equivocal results on IHC (2+) from July 2010 to September 2013 were studied. Expression of HER2/neu IHC and FISH was analyzed.

Fluorescent in situ hybridization

FISH analysis was performed using the PathVysion HER2 probe kit (Vysis, USA). All procedures were followed according to the HER2 probe kit instruction manual (Vysis, USA). The

\begin{tabular}{|l|}
\hline Access this article online \\
\hline Quick Response Code: \\
\\
\hline Website: www.sajc.org \\
\hline DOI: $10.4103 / 2278-330 X .208841$ \\
\hline
\end{tabular}

Uhiversiong of Bioengineering and Technology, Gauh University, Guwahati, Assam, 'Rajiv Gandhi Cancer Institute and Research Centre, New Delhi, India Correspondence to: Mr. Manoj Kumar Panigrahi, E-mail: manojkumar.panigrahi8I@gmail.com slides were screened under fluorescent microscope (Leica $6000 \mathrm{M}$, Germany) using appropriate filters.

\section{Scoring methods}

Scoring methods for IHC used according to 2007 and FISH were done according to the ASCO/CAP guidelines 2007 and 2013.

\section{Statistical analysis}

A nonparametric Chi-square statistics test was used for testing associations between variables and $P<0.05$ were considered statistically significant. Statistical analysis was performed using the Statistical Package for Social Sciences software (version 22.0; SPSS Inc., Chicago, IL, USA).

\section{Results}

One hundred and ninety-two subjects (34.4\%) were HER2 amplified according to CAP scoring 2007. Moreover, on reanalysis according to 2013 CAP scoring criteria, we found 246 subjects (44\%) HER2 amplified. We also found 106 cases $(19 \%)$ polysomy 17 which showed $24(22.6 \%)$ cases of HER2 amplification according to CAP 2007 scoring criteria and 36 (33.9\%) cases according to 2013 CAP scoring.

\section{Discussion}

Using both 2007 and 2013 ASCO/CAP scoring method on FISH technique, we found $10 \%$ of difference between two methods of scoring. Using FISH, we also investigated the frequency of polysomy 17 and its association with HER2 alteration in patients with invasive breast cancer. As polysomy 17 are relatively common in breast carcinoma, it is possible that HER2 FISH results can be misinterpreted. Vanden Bempt et al. reported that $>40 \%$ of breast carcinomas with polysomy $17^{[5]}$ and $\mathrm{Pu}$ et al. reported $57.1 \%$ breast carcinoma with polysomy $17 .{ }^{[6]}$ However, in the Indian population, our study

This is an open access article distributed under the terms of the Creative Commons Attribution-NonCommercial-ShareAlike 3.0 License, which allows others to remix, tweak, and build upon the work non-commercially, as long as the author is credited and the new creations are licensed under the identical terms.
For reprints contact: reprints@ medknow.com

How to cite this article: Panigrahi MK, Kumar D, Mehta A, Saikia KK. Outcome of HER2 Testing by FISH applying ASCO/CAP 2007 and 2013 guideline in IHC equivocal group of breast cancer: Experience at tertiary cancer care centre. South Asian J Cancer 2017;6:45-6. 
showed $19 \%$ breast carcinoma with polysomy 17 . When we applied 2013 CAP scoring criteria, we found 14.6\% increase in number for HER2 amplification with polysomy 17. As we shown one case in Figure 1, polysomy17 with negative according to CAP 2007 guideline but when we applied 2013 CAP guideline on the same case, the case should be given positive with polysomy. When we re-evaluated the 82 cases that were HER2 nonamplified and polysomy 17 accompanied, we found that $12(14.6 \%)$ cases could be defined as HER2 amplified. These $14.6 \%$ patients showed HER2 amplification may be suitable for anti-HER2 targeted therapy. There was a significant increase in the number of HER2 amplified cases, i.e., cases increase from 192 to 246 out of 557 cases. As recent study suggested that the presence of CEP17 alterations could identify a more aggressive subset of breast cancers that are nonresponsive to conventional therapy independently of HER2 amplification status. ${ }^{[7]}$ Some researchers believe that polysomy 17 without HER2 amplification do not predict response to lapatinib in metastatic breast cancer. ${ }^{[7]}$

\section{Conclusion}

FISH results were evaluated (IHC2+ interpreted according to CAP 2007 guideline) with both 2007 and $2013 \mathrm{ASCO} /$ CAP scoring criteria, we identified significantly more HER2 positive cases as compared to cases evaluated using the 2007 criteria $(P<0.05)$. Polysomy of CEP17 is a crucial cause of misinterpretation of HER2 FISH result. Using the 2013 ASCO/ CAP scoring criteria on $\mathrm{IHC} 2+$ cases evaluate significantly higher in a number of HER2 amplification with polysomy of chromosome 17, which shows more patients appropriate for targeted treatment.

\section{Acknowledgments}

The author acknowledges the patients for sharing the clinical information and samples, the oncologists for referring the patients for the HER2/neu testing and the management

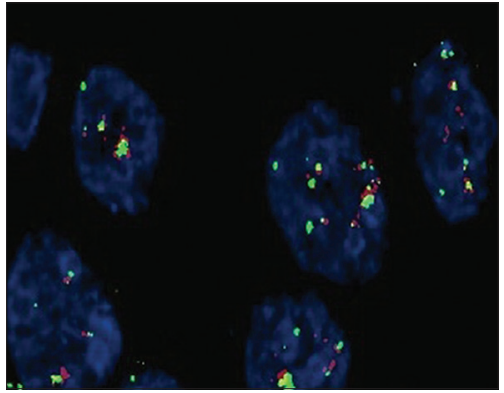

Figure 1: Polysomy with negative (CAP 2007)

of Rajiv Gandhi Cancer Institute and Research Centre, New Delhi, India.

Financial support and sponsorship

Nil.

\section{Conflicts of interest}

There are no conflicts of interest.

\section{References}

1. National Cancer Institute. SEER Cancer Statistics Review 2. Female Breast Cancer (Invasive): Age-Adjusted SEER Incidence and US Death Rates by Race/Ethnicity and Sex. 2012. Available from: http://www.seer. cancer.gov/csr/1975_2001/results_merged/topic_race_ethnicity.pdf.

2. Paterson MC, Dietrich KD, Danyluk J, Paterson AH, Lees AW, Jamil N, et al. Correlation between c-erbB-2 amplification and risk of recurrent disease in node-negative breast cancer. Cancer Res 1991;51:556-67.

3. Hyun CL, Lee HE, Kim KS, Kim SW, Kim JH, Choe G, et al. The effect of chromosome 17 polysomy on HER-2/neu status in breast cancer. J Clin Pathol 2008;61:317-21.

4. Rosenberg CL. Polysomy 17 and HER-2 amplification: True, true, and unrelated. J Clin Oncol 2008;26:4856-8.

5. Vanden Bempt I, Van Loo P, Drijkoningen M, Neven P, Smeets A, Christiaens MR, et al. Polysomy 17 in breast cancer: Clinicopathologic significance and impact on HER-2 testing. J Clin Oncol 2008;26:4869-74.

6. Pu X, Shi J, Li Z, Feng A, Ye Q. Comparison of the 2007 and 2013 ASCO/ CAP evaluation systems for HER2 amplification in breast cancer. Pathol Res Pract 2015;211:421-5.

7. Watters AD, Going JJ, Cooke TG, Bartlett JM. Chromosome 17 aneusomy is associated with poor prognostic factors in invasive breast carcinoma. Breast Cancer Res Treat 2003;77:109-14. 